\title{
Erratum to: Editorial for a special issue on kidney transplant
}

\author{
Oscar Rodriguez Faba · Alberto Breda
}

Published online: 18 April 2014

(C) Springer-Verlag Berlin Heidelberg 2014

\section{Erratum to: World J Urol \\ DOI 10.1007/s00345-013-1101-8}

This editorial was intended for publication in the same issue as a collection of articles on kidney transplant (World Journal of Urology volume 31, issue 4; listed below). Owing to an oversight, however, it was not included. The editors and publisher of World Journal of Urology would like to apologize for this error.

Hellegering J, Visser J, Kloke HJ, D'Ancona FC, Hoitsma AJ, van der Vliet JA, Warle MC (2013) Poor early graft function impairs long-term outcome in living donor kidney transplantation. World J Urol 31:901-906. doi:10.1007/ s00345-012-0835-z

Friedersdorff F, Roller C, Klein G, Werthemann P, Cash H, Budde K, Slowinski T, Kempkensteffen C, Busch J, Fuller TF et al (2013) Outcome of expanded criteria donor kidneys that were transplanted at other Eurotransplant centers after being rejected by our institution. World J Urol 31:947-952. doi:10.1007/s00345-012-0929-7

The online version of the original article can be found under doi:10.1007/s00345-013-1101-8.

O. Rodriguez Faba $(\varangle) \cdot$ A. Breda

Fundació Puigvert, C/Cartagena, 340-350, 08025 Barcelona,

Spain

e-mail: orodriguez@fundacio-puigvert.es

A. Breda

e-mail: albbred@hotmail.com
Lucarelli G, Vavallo A, Bettocchi C, Losappio V, Gesualdo L, Grandaliano G, Selvaggi FP, Battaglia M, Ditonno P (2013) Impact of transplant nephrectomy on retransplantation: a single-center retrospective study. World J Urol 31:959-963. doi:10.1007/s00345-012-0986-y

Cosentino M, Breda A, Sanguedolce F, Landman J, Stolzenburg JU, Verze P, Rassweiler J, Van Poppel H, Klingler HC, Janetschek G et al (2013) The use of mannitol in partial and live donor nephrectomy: an international survey. World J Urol 31:977-982. doi:10.1007/s00345-012-1003-1 